\title{
Subclasses of Starlike Functions Associated with Fractional $q$-Calculus Operators
}

\author{
G. Murugusundaramoorthy, ${ }^{1}$ C. Selvaraj, ${ }^{2}$ and O. S. Babu ${ }^{3}$ \\ ${ }^{1}$ School of Advanced Sciences, VIT University, Vellore 632014, India \\ ${ }^{2}$ Department of Mathematics, Presidency College (Autonomous), Chennai 600005, India \\ ${ }^{3}$ Department of Mathematics, Dr. Ambedkar Government Arts College, Chennai 600039, India
}

Correspondence should be addressed to G. Murugusundaramoorthy; gmsmoorthy@yahoo.com

Received 3 January 2013; Accepted 27 March 2013

Academic Editor: Vladislav Kravchenko

Copyright (C) 2013 G. Murugusundaramoorthy et al. This is an open access article distributed under the Creative Commons Attribution License, which permits unrestricted use, distribution, and reproduction in any medium, provided the original work is properly cited.

\begin{abstract}
Making use of fractional $q$-calculus operators, we introduce a new subclass $\mathscr{M}_{q}(\lambda, \gamma, k)$ of starlike functions and determine the coefficient estimate, extreme points, closure theorem, and distortion bounds for functions in $\mathscr{M}_{q}(\lambda, \gamma, k)$. Furthermore we discuss neighborhood results, subordination theorem, partial sums, and integral means inequalities for functions in $\mathscr{M}_{q}(\lambda, \gamma, k)$.
\end{abstract}

\section{Introduction and Preliminaries}

Denote by $\mathscr{A}$ the class of functions of the form

$$
f(z)=z+\sum_{n=2}^{\infty} a_{n} z^{n}
$$

which are analytic and univalent in the open $\operatorname{disc} U=\{z$ : $|z|<1\}$ and normalized by $f(0)=0=f^{\prime}(0)-1$. Due to Silverman [1], denote by $\mathscr{T}$ a subclass of $\mathscr{A}$ consisting of functions of the form

$$
f(z)=z-\sum_{n=2}^{\infty} a_{n} z^{n}, \quad a_{n} \geq 0, z \in U .
$$

The fractional calculus operator has gained importance and popularly due to vast potential demonstrated applications in various fields of science, engineering and also in the geometric function theory. The fractional $q$-calculus operator is the extension of the ordinary fractional calculus in the $q$ theory. Recently Purohit and Raina [2] investigated applications of fractional $q$-calculus operator to define new classes of functions which are analytic in the open unit disc. We recall the definitions of fractional $q$-calculus operators of complex valued function $f(z)$.
The $q$-shifted factorial is defined for $\alpha, q \in \mathbb{C}$ as a product of $n$ factors by

$$
(\alpha ; q)_{n}= \begin{cases}1, & n=0, \\ (1-\alpha)(1-\alpha q) \cdots\left(1-\alpha q^{n-1}\right), & n \in N,\end{cases}
$$

and in terms of basic analogue of the gamma function

$$
\left(q^{\alpha} ; q\right)_{n}=\frac{\Gamma_{q}(\alpha+n)(1-q)^{n}}{\Gamma_{q}(\alpha)}, \quad n>0 .
$$

Due to Gasper and Rahman [3], the recurrence relation for $q$-gamma function is given by

$$
\Gamma_{q}(1+\alpha)=\frac{\left(1-q^{\alpha}\right) \Gamma_{q}(\alpha)}{1-q}
$$

and the $q$ binomial expansion is given by

$$
\begin{aligned}
(x-y)_{v} & =x^{v}\left(-\frac{y}{x} ; q\right)=x^{\nu} \prod_{n=0}^{\infty} \frac{1-(y / x) q^{n}}{1-(y / x) q^{v+n}} \\
& =x_{1}^{v} \Phi_{0}\left[q^{-v} ;-; q, \frac{y q^{v}}{x}\right] .
\end{aligned}
$$


Further the $q$-derivative and $q$-integral of functions $f$ defined on the subset of $\mathbb{C}$ are, respectively, given by

$$
\begin{gathered}
D_{q, z} f(z)=\frac{f(z)-f(z q)}{z(1-q)}, \quad(z \neq 0, \quad q \neq 0), \\
\int_{0}^{z} f(t) d(t ; q)=z(1-q) \sum_{k=0}^{\infty} q^{k} f\left(z q^{k}\right) .
\end{gathered}
$$

It is interest to note that $\lim _{q \rightarrow 1^{-}}\left(\left(q^{\alpha} ; q\right)_{n} /(1-q)^{n}\right)=(\alpha)_{n}=$ $\alpha(\alpha+1) \cdots(\alpha+n-1)$ the familiar Pochhammer symbol. Due to Kim and Srivastava [4], we recall the following definitions of fractional $q$-integral and fractional $q$-derivative operators, which are very much useful for our study.

Definition 1. Let the function $f(z)$ be analytic in a simply connected region of the $z$-plane containing the origin. The fractional $q$-integral of $f$ of order $\mu$ is defined by

$$
\begin{aligned}
\mathscr{I}_{q, z}^{\mu} f(z) & =\mathscr{D}_{q, z}^{-\mu} f(z) \\
& =\frac{1}{\Gamma_{q}(\mu)} \int_{0}^{z}(z-q t)_{\mu-1} f(t) d(t ; q), \quad \mu>0,
\end{aligned}
$$

where $(z-t q)_{\mu-1}$ can be expressed as the $q$-binomial given by (6) and the series ${ }_{1} \Phi_{0}[\mu ;-; q, z]$ is a single valued when $|\arg (z)|<\pi$ and $|z|<1$, therefore the function $(z-t q)_{\mu-1}$ in (8) is single valued when $\left|\arg \left(-t q^{\mu} / z\right)\right|<\pi$, $\left|t q^{\mu}\right|<1$ and $|\arg (z)|<\pi$.

Definition 2. The fractional $q$-derivative operator of order $\mu$ is defined for a function $f(z)$ by

$$
\begin{aligned}
\mathscr{D}_{q, z}^{\mu} f(z)= & \mathscr{D}_{q, z} \mathcal{J}_{q, z}^{1-\mu} f(z) \\
= & \frac{1}{\Gamma_{q}(1-\mu)} D_{q, z} \\
& \times \int_{0}^{z}(z-q t)_{-\mu} f(t) d(t ; q), \quad 0 \leq \mu<1,
\end{aligned}
$$

where the function $f(z)$ is constrained, and the multiplicity of the function $(z-t q)_{-\mu}$ is removed as in Definition 1 .

Definition 3. Under the hypothesis of Definition 2, the fractional derivative of order $\mu$ is defined by

$$
D_{q, z}^{\mu} f(z)=D_{q, z}^{m} \mathcal{J}_{q, z}^{m-\mu} f(z), \quad\left(m-1 \leq \mu<m ; m \in N_{0}\right) .
$$

With the aid of the above definitions, and their known extensions involving $q$-differintegral operator we define the linear operator $\Omega_{q, z}^{\mu} f(z): \mathscr{T} \rightarrow \mathscr{T}$

$$
\begin{aligned}
\Omega_{q, z}^{\mu} f(z) & =\frac{\Gamma_{q}(2-\mu)}{\Gamma_{q}(2)} z^{\mu-1} D_{q, z}^{\mu} f(z) \\
& =z-\sum_{n=2}^{\infty} \Phi_{q}(n, \mu) a_{n} z^{n},
\end{aligned}
$$

where

$$
\Phi_{q}(n, \mu)=\frac{\Gamma_{q}(2-\mu) \Gamma_{q}(n+1)}{\Gamma_{q}(2) \Gamma_{q}(n+1-\mu)},
$$

where $\mu<2,0<q<1$ and $z \in U$. Here $D_{q, z}^{\mu} f(z)$ in (10) represents, respectively, a fractional $q$-integral of $f(z)$ of order $\mu$ when $-\infty<\mu<0$ and fractional $q$-derivative of $f(z)$ of order $\mu$ when $0 \leq \mu<2$.

In 1975, Silverman [1] studied two interesting subclasses of $f(z) \in \mathscr{T}$, namely, $\mathscr{T}^{*}(\gamma)$, the class of starlike function of order $\gamma(0 \leq \gamma<1)$ if $\mathfrak{R}\left(z f^{\prime}(z) / f(z)\right)>\gamma$ and $C(\gamma)$, the class of convex function of order $\gamma(0 \leq \gamma<1)$ if $\mathfrak{R}\left(1+\left(z f^{\prime \prime}(z) / f^{\prime}(z)\right)\right)>\gamma$. Motivated by the earlier works of Goodman [5] and Rønning [6, 7] in this paper we define the following new subclass of $k$-starlike functions of order $\gamma$ based on the $q$-fractional operator.

For $0 \leq \lambda<1,0 \leq \gamma<1, \mu<2$, and $k \geq 0$, we let $\mathscr{M}_{q}(\lambda$, $\gamma, k)$ be the subclass of $T$ consisting of functions of the form (2) and satisfying the analytic criterion

$$
\begin{aligned}
& \Re\left(\frac{\left(\Omega_{q, z}^{\mu} f(z)\right)^{\prime}+\lambda z\left(\Omega_{q, z}^{\mu} f(z)\right)^{\prime \prime}}{\left(\Omega_{q, z}^{\mu} f(z)\right)^{\prime}+z\left(\Omega_{q, z}^{\mu} f(z)\right)^{\prime \prime}}-\gamma\right) \\
& \quad>k\left|\frac{\left(\Omega_{q, z}^{\mu} f(z)\right)^{\prime}+\lambda z\left(\Omega_{q, z}^{\mu} f(z)\right)^{\prime \prime}}{\left(\Omega_{q, z}^{\mu} f(z)\right)^{\prime}+z\left(\Omega_{q, z}^{\mu} f(z)\right)^{\prime \prime}}-1\right|
\end{aligned}
$$

$z \in U$, and $\Omega_{q, z}^{\mu} f(z)$ is given by (11).

For different choices of $\lambda$ we state some special cases of subclasses of $\mathscr{M}_{q}(\lambda, \gamma, k)$ as illustrated in the following examples.

Example 4. For $\lambda=0$, we let

$$
\begin{aligned}
\mathscr{U} \mathscr{C} \mathscr{M}_{q}(\gamma, k) & :=\left\{f \in \mathscr{T}: \mathfrak{R}\left(\frac{\left(\Omega_{q, z}^{\mu} f(z)\right)^{\prime}}{\left(\Omega_{q, z}^{\mu} f(z)\right)^{\prime}+z\left(\Omega_{q, z}^{\mu} f(z)\right)^{\prime \prime}}-\gamma\right)\right. \\
> & \left.k\left|\frac{\left(\Omega_{q, z}^{\mu} f(z)\right)^{\prime}}{\left(\Omega_{q, z}^{\mu} f(z)\right)^{\prime}+z\left(\Omega_{q, z}^{\mu} f(z)\right)^{\prime \prime}}-1\right|\right\},
\end{aligned}
$$

where $0 \leq \gamma<1, \mu<2, k \geq 0$, and $z \in U$.

Example 5. For $\lambda=0$ and $k=0$, we let

$$
\begin{aligned}
& \mathscr{M T}_{q}(\gamma) \\
& :=\left\{f \in \mathscr{T}: \mathfrak{R}\left(\frac{\left(\Omega_{q, z}^{\mu} f(z)\right)^{\prime}}{\left(\Omega_{q, z}^{\mu} f(z)\right)^{\prime}+z\left(\Omega_{q, z}^{\mu} f(z)\right)^{\prime \prime}}\right)>\gamma,\right. \\
& z \in U\},
\end{aligned}
$$

where $0 \leq \gamma<1, \mu<2$ and $z \in U$. 
In this paper we determine the coefficient estimate, extreme points, closure theorem, and distortion bounds for functions in $\mathscr{M}_{q}(\lambda, \gamma, k)$. Furthermore we discuss neighborhood results, subordination theorem, partial sums, and integral means inequalities for functions in $\mathscr{M}_{q}(\lambda, \gamma, k)$.

\section{Characterization Properties of $\mathscr{M}_{q}(\lambda, \gamma, k)$}

We recall the following lemmas to prove our main results.

Lemma 6. If $\gamma$ is a real number and $w$ is a complex number, then $\mathfrak{R}(w) \geq \gamma \Leftrightarrow|w+(1-\gamma)|-|w-(1+\gamma)| \geq 0$.

Lemma 7. If $w$ is a complex number and $\gamma$, $k$ are real numbers, then

$$
\begin{array}{r}
\mathfrak{R}(w) \geq k|w-1|+\gamma \Longleftrightarrow \mathfrak{R}\left\{w\left(1+k e^{i \theta}\right)-k e^{i \theta}\right\} \geq \gamma, \\
-\pi \leq \theta \leq \pi .
\end{array}
$$

Lemma 8. Let $0 \leq \lambda<1,0 \leq \gamma<1, k \geq 0$ then a function $f \in \mathscr{M}_{q}(\lambda, \gamma, k)$ if and only if

$$
\sum_{n=2}^{\infty} n[(1+n \lambda-\lambda)(1+k)-n(\gamma+k)] \Phi_{q}(n, \mu)\left|a_{n}\right| \leq 1-\gamma
$$

where $\Phi_{q}(n, \mu)$ is given by (12).

Proof. Let a function $f$ of the form (2) in $T$ satisfy the condition (17). We will show that (13) is satisfied, and so $f \in \mathscr{M}_{q}(\lambda, \gamma, k)$. Using Lemma 7 , it is enough to show that

$$
\begin{array}{r}
\Re\left(\frac{\left(\Omega_{q, z}^{\mu} f(z)\right)^{\prime}+\lambda z\left(\Omega_{q, z}^{\mu} f(z)\right)^{\prime \prime}}{\left(\Omega_{q, z}^{\mu} f(z)\right)^{\prime}+z\left(\Omega_{q, z}^{\mu} f(z)\right)^{\prime \prime}}\left(1+k e^{i \theta}\right)-k e^{i \theta}\right)>\gamma, \\
-\pi \leq \theta \leq \pi .
\end{array}
$$

That is, suppose $f \in \mathscr{M}_{q}(\lambda, \gamma, k)$, then by Lemma 7 and by choosing the values of $z$ on the positive real axis inequality (18) reduces to

$$
\begin{aligned}
& \Re((1-\gamma) \\
& -\sum_{n=2}^{\infty} n\left[\left(1+k e^{i \theta}\right)(1+\lambda n-\lambda)-n\left(\gamma+k e^{i \theta}\right)\right] \\
& \left.\times \Phi_{q}(n, \mu)\left|a_{n}\right| z^{n-1}\right) \\
& \left.\times\left(1-\sum_{n=2}^{\infty} n^{2} \Phi_{q}(n, \mu) a_{n} z^{n-1}\right)^{-1}\right) \geq 0 .
\end{aligned}
$$

Since $\operatorname{Re}\left(-e^{i \theta}\right) \geq-e^{i 0}=-1$, the previous inequality reduces to

$$
\begin{aligned}
& \mathfrak{R}((1-\gamma) \\
& -\sum_{n=2}^{\infty} n[(k+1)(1+n \lambda-\lambda)-n(\gamma+k)] \\
& \left.\quad \times \Phi_{q}(n, \mu) a_{n} r^{n-1}\right) \\
& \left.\times\left(1-\sum_{n=2}^{\infty} n^{2} \Phi_{q}(n, \mu) a_{n} r^{n-1}\right)^{-1}\right) \geq 0 .
\end{aligned}
$$

Letting $r \rightarrow 1^{-}$and by the mean value theorem we get desired inequality (17).

Conversely, let (17) hold; we will show that (13) is satisfied, and so $f \in \mathscr{M}_{q}(\lambda, \gamma, k)$. In view of Lemma $6, \mathfrak{R}(w)>\gamma \Leftrightarrow$ $|w-(1+\gamma)|<|w+(1-\gamma)|$, it is enough to show that

$$
\begin{aligned}
& \left|\frac{A(z)}{B(z)}-\left(1+k\left|\frac{A(z)}{B(z)}-1\right|+\gamma\right)\right| \\
& \quad<\left|\frac{A(z)}{B(z)}+\left(1-k\left|\frac{A(z)}{B(z)}-1\right|-\gamma\right)\right|,
\end{aligned}
$$

where

$$
\begin{aligned}
A(z) & :=\left(\Omega_{q, z}^{\mu} f(z)\right)^{\prime}+\lambda z\left(\Omega_{q, z}^{\mu} f(z)\right)^{\prime \prime} \\
& =1-\sum_{n=2}^{\infty} n(1+n \lambda-\lambda) \Phi_{q}(n, \mu)\left|a_{n}\right| z^{n-1}, \\
B(z) & :=\left(\Omega_{q, z}^{\mu} f(z)\right)^{\prime}+z\left(\Omega_{q, z}^{\mu} f(z)\right)^{\prime \prime} \\
& =1-\sum_{n=2}^{\infty} n^{2} \Phi_{q}(n, \mu)\left|a_{n}\right| z^{n-1} .
\end{aligned}
$$

Hence, one has

$$
\begin{aligned}
L= & \left|\frac{A(z)}{B(z)}-\left(1+k\left|\frac{A(z)}{B(z)}-1\right|+\gamma\right)\right| \\
< & \frac{1}{|B(z)|} \\
& \times \mid-\gamma-\sum_{n=2}^{\infty}\left[n(1+n \lambda-\lambda)(1+k)-n^{2}(1+\gamma+k)\right] \\
& \quad \times \Phi_{q}(n, \mu) a_{n} z^{n-1} \mid \\
<\frac{1}{|B(z)|} \mid(2-\gamma) & \quad-\sum_{n=2}^{\infty}\left[n(1+n \lambda-\lambda)(1+k)+n^{2}(1-\gamma-k)\right] \\
< & \quad \times \quad \Phi_{q}(n, \mu) a_{n} z^{n-1} \mid \\
& \left|\frac{A(z)}{B(z)}+\left(1-k\left|\frac{A(z)}{B(z)}-1\right|-\gamma\right)\right|
\end{aligned}
$$


and it is easy to show that $R-L>0$, by the given condition (17), and the proof is complete.

Corollary 9. If $f \in \mathscr{M}_{q}(\lambda, \gamma, k)$, then $\left|a_{n}\right| \leq(1-\gamma) / \Psi(\lambda$, $\gamma, k, n), 0 \leq \lambda \leq 1,0 \leq \gamma<1, k \geq 0$, where $\Psi(\lambda, \gamma, k, n)=$ $n[(1+n \lambda-\lambda)(1+k)-(\gamma+k)] \Phi_{q}(n, \mu)$. Equality holds for the function $f(z)=z-((1-\gamma) / \Psi(\lambda, \gamma, k, n)) z^{n}$.

For the sake of brevity we let

(i) $\Psi(\lambda, \gamma, k, n)$

$$
=n[(1+n \lambda-\lambda)(1+k)-n(\gamma+k)] \Phi_{q}(n, \mu),
$$

(ii) $\Psi(\lambda, \gamma, k, 2)=2[(1+\lambda)(k+1)-2(\gamma+k)] \Phi_{q}(2, \mu)$,

(iii) $\Phi_{q}(2, \mu)=\frac{\Gamma_{q}(2-\mu) \Gamma_{q}(3)}{\Gamma_{q}(2) \Gamma_{q}(3-\mu)}=\frac{\left(1-q^{2}\right)}{\left(1-q^{2-\mu}\right)}$,

unless otherwise stated.

Remark 10. First we show that the function

$$
\Phi_{q}(n, \mu)=\frac{\Gamma_{q}(2-\mu) \Gamma_{q}(n+1)}{\Gamma_{q}(2) \Gamma_{q}(n+1-\mu)}, \quad n \geq 2
$$

is a decreasing function of $n$ for $\mu<2,0<q<1$. It follows that

$$
\frac{\Phi_{q}(n+1, \mu)}{\Phi_{q}(n, \mu)}=\frac{\Gamma_{q}(n+1-\mu) \Gamma_{q}(n+2)}{\Gamma_{q}(n+1) \Gamma_{q}(n+2-\mu)}
$$

and it is sufficient to consider here the value $n<2$, so that on using (5) we get

$$
\frac{\Phi_{q}(3, \mu)}{\Phi_{q}(2, \mu)}=\frac{1-q^{3}}{1-q^{3-\mu}}, \quad 0<q<1 .
$$

The function $\Phi_{q}(n, \mu)$ is a decreasing function of $n$ if $\Phi_{q}(3$, $\mu) / \Phi_{q}(2, \mu) \leq 1$, and this gives $\left(1-q^{3}\right) /\left(1-q^{3-\mu}\right) \leq 1$. Multiplying the previous inequality both sides by $1-q^{3-\mu}$ provided $\mu<2$; we are at once lead to the inequality $\mu \leq 0$. Thus, $\Phi_{q}(n, \mu)$ is a decreasing function of $n$ for $-\infty<\mu<2,0<$ $q<1$.

Now by routine procedure using the techniques employed by Silverman [1] we can easily prove the following theorems.

Theorem 11. Let the function $f(z)$ defined by (2) belong to $\mathscr{M}_{q}(\lambda, \gamma, k)$, then

$$
\begin{aligned}
& r-\frac{1-\gamma}{\Psi(\lambda, \gamma, k, 2)} r^{2} \\
& \quad \leq|f(z)| \leq r+\frac{1-\gamma}{\Psi(\lambda, \gamma, k, 2)} r^{2}, \quad|z|=r, \\
& 1-\frac{2(1-\gamma)}{\Psi(\lambda, \gamma, k, 2)} r \\
& \quad \leq\left|f^{\prime}(z)\right| \leq 1+\frac{2(1-\gamma)}{\Psi(\lambda, \gamma, k, 2)} r, \quad|z|=r .
\end{aligned}
$$

Equalities are sharp for the function $f(z)=z-((1-\gamma) / \Psi(\lambda$, $\gamma, k, 2)) z^{2}$, where $\Psi(\lambda, \gamma, k, 2)$ is obtained from (25).

Theorem 12 (extreme points). The extreme points of $\mathscr{M}_{q}(\lambda$, $\gamma, k)$ are $f_{1}(z)=z$ and $f_{n}(z)=z-((1-\gamma) / \Psi(\lambda, \gamma, k, n)) z^{n}$, for $n=2,3,4, \ldots$ Then $f \in M_{q}(\lambda, \gamma, k)$ if and only if it can be expressed in the form $f(z)=\sum_{n=1}^{\infty} \omega_{n} f_{n}(z),\left(\omega_{n} \geq 0\right)$, $\sum_{n=1}^{\infty} \omega_{n}=1$, where $\Psi(\lambda, \gamma, k, n)$ is defined in $(24)$.

Theorem 13. Let the functions $f_{j}(z)(j=1,2, \ldots, m)$ defined by

$$
f_{j}(z)=z-\sum_{n=2}^{\infty} a_{n, j} z^{n} \quad \text { for } a_{n, j} \geq 0, z \in U \text {. }
$$

be in the classes $\mathscr{M}_{q}\left(\lambda, \gamma_{j}, k\right)(j=1,2, \ldots, m)$, respectively. Then the function $h(z)=z-(1 / m) \sum_{n=2}^{\infty}\left(\sum_{j=1}^{m} a_{n, j}\right) z^{n}$ is in the class $\mathscr{M}_{q}(\lambda, \gamma, k)$, where $\gamma=\min _{1 \leq j \leq m}\left\{\gamma_{j}\right\}\left(-1 \leq \gamma_{j}<1\right)$.

\section{Neighbourhood Results}

In this section, we discuss neighbourhood results of the class $\mathscr{M}_{q}(\lambda, \gamma, k)$. Following $[8,9]$, we define the $\delta$-neighbourhood of function $f(z) \in \mathscr{T}$ by

$$
\begin{aligned}
N_{\delta}(f):= & \left\{h \in \mathscr{T}: h(z)=z-\sum_{n=2}^{\infty} d_{n} z^{n},\right. \\
& \left.\sum_{n=2}^{\infty} n\left|a_{n}-d_{n}\right| \leq \delta\right\} .
\end{aligned}
$$

Particularly for the identity function $e(z)=z$, we have

$$
\begin{aligned}
N_{\delta}(e):= & \left\{h \in \mathscr{T}: h(z)=z-\sum_{n=2}^{\infty} d_{n} z^{n},\right. \\
& \left.\sum_{n=2}^{\infty} n\left|d_{n}\right| \leq \delta\right\} .
\end{aligned}
$$

Theorem 14. If

$$
\delta:=\frac{1-\gamma}{[(1+\lambda)(1+k)-2(\gamma+k)] \Phi_{q}(2, \mu)}
$$

then $\mathscr{M}_{q}(\lambda, \gamma, k) \subset N_{\delta}(e)$, where $\Psi(\lambda, \gamma, k, 2)$ is defined in (25).

Proof. For $f \in \mathscr{M}_{q}(\lambda, \gamma, k)$, Lemma 8 immediately yields

$$
\Psi(\lambda, \gamma, k, 2) \sum_{n=2}^{\infty} a_{n} \leq 1-\gamma,
$$

so that

$$
\sum_{n=2}^{\infty} a_{n} \leq \frac{1-\gamma}{\Psi(\lambda, \gamma, k, 2)} .
$$

On the other hand, we find from (17) and (36) that

$$
\sum_{n=2}^{\infty} n a_{n} \leq \frac{1-\gamma}{[(1+\lambda)(1+k)-2(\gamma+k)] \Phi_{q}(2, \mu)},
$$

which, in view of the definition (33), proves Theorem 14. 
Now we determine the neighborhood for the class $\mathscr{M}_{q}(\rho$, $\lambda, \gamma, k)$ which we define as follows. A function $f \in \mathscr{T}$ is said to be in the class $\mathscr{M}_{q}(\rho, \lambda, \gamma, k)$ if there exists a function $h \in$ $\mathscr{M}_{q}(\rho, \lambda, \gamma, k)$ such that

$$
\left|\frac{f(z)}{h(z)}-1\right|<1-\rho, \quad(z \in U, 0 \leq \rho<1) .
$$

Theorem 15. If $h \in \mathscr{M}_{q}(\rho, \lambda, \gamma, k)$ and

$$
\rho=1-\frac{\delta \Psi(\lambda, \gamma, k, 2)}{2[\Psi(\lambda, \gamma, k, 2)-(1-\gamma)]}
$$

then

$$
N_{\delta}(h) \subset \mathscr{M}_{q}(\rho, \lambda, \gamma, k),
$$

where $\Psi(\lambda, \gamma, k, 2)$ is defined in (25).

Proof. Suppose that $f \in N_{\delta}(h)$; we then find from (32) that

$$
\sum_{n=2}^{\infty} n\left|a_{n}-d_{n}\right| \leq \delta
$$

which yields

$$
\sum_{n=2}^{\infty}\left|a_{n}-d_{n}\right| \leq \frac{\delta}{2} .
$$

Next, since $h \in \mathscr{M}_{q}(\lambda, \gamma, k)$, we have

$$
\sum_{n=2}^{\infty} d_{n}=\frac{1-\gamma}{\Psi(\lambda, \gamma, k, 2)}
$$

so that

$$
\begin{aligned}
\left|\frac{f(z)}{h(z)}-1\right| & <\frac{\sum_{n=2}^{\infty}\left|a_{n}-d_{n}\right|}{1-\sum_{n=2}^{\infty} d_{n}} \\
& \leq \frac{\delta}{2} \times \frac{\Psi(\lambda, \gamma, k, 2)}{\Psi(\lambda, \gamma, k, 2)-(1-\gamma)} \\
& \leq \frac{\delta \Psi(\lambda, \gamma, k, 2)}{2[\Psi(\lambda, \gamma, k, 2)-(1-\gamma)]} \\
& =1-\rho
\end{aligned}
$$

provided that $\rho$ is given precisely by (39). Thus $f \in \mathscr{M}_{q}(\rho, \lambda$, $\gamma, k$ ) for $\rho$ given by (39), completes the proof.

\section{Subordination Results}

Now we recall the following results due to Wilf [10], which are very much needed for our study.

Definition 16 (subordination principle [11]). For analytic functions $g$ and $h$ with $g(0)=h(0), g$ is said to be subordinate to $h$, denoted by $g<h$, if there exists an analytic function $w$ such that $w(0)=0,|w(z)|<1$, and $g(z)=h(w(z))$, for all $z \in U$.
Definition 17 (subordinating factor sequence). A sequence $\left\{b_{n}\right\}_{n=1}^{\infty}$ of complex numbers is said to be a subordinating sequence if, whenever $f(z)=\sum_{n=1}^{\infty} a_{n} z^{n}, a_{1}=1$ is regular, univalent, and convex in $U$, we have

$$
\sum_{n=1}^{\infty} b_{n} a_{n} z^{n}<f(z), \quad z \in U .
$$

Lemma 18. The sequence $\left\{b_{n}\right\}_{n=1}^{\infty}$ is a subordinating factor sequence if and only if

$$
\operatorname{Re}\left\{1+2 \sum_{n=1}^{\infty} b_{n} z^{n}\right\}>0, \quad z \in U .
$$

Theorem 19. Let $f \in \mathscr{M}_{q}(\lambda, \gamma, k)$ and $g(z)$ be any function in the usual class of convex functions $C$, then

$$
\frac{\Psi(\lambda, \gamma, k, 2)}{2[1-\gamma+\Psi(\lambda, \gamma, k, 2)]}(f * g)(z) \prec g(z),
$$

where $0 \leq \gamma<1, k \geq 0,0 \leq \lambda \leq 1$, and

$$
\operatorname{Re}\{f(z)\}>-\frac{[1-\gamma+\Psi(\lambda, \gamma, k, 2)]}{\Psi(\lambda, \gamma, k, 2)}, \quad z \in U .
$$

The constant factor $\Psi(\lambda, \gamma, k, 2) / 2[1-\gamma+\Psi(\lambda, \gamma, k, 2)]$ in (47) cannot be replaced by a larger number.

Proof. Let $f \in \mathscr{M}_{q}(\lambda, \gamma, k)$, and suppose that $g(z)=z+$ $\sum_{n=2}^{\infty} b_{n} z^{n} \in C$. Then

$$
\begin{aligned}
& \frac{\Psi(\lambda, \gamma, k, 2)}{2[1-\gamma+\Psi(\lambda, \gamma, k, 2)]}(f * g)(z) \\
& \quad=\frac{\Psi(\lambda, \gamma, k, 2)}{2[1-\gamma+\Psi(\lambda, \gamma, k, 2)]}\left(z+\sum_{n=2}^{\infty} b_{n} a_{n} z^{n}\right) .
\end{aligned}
$$

Thus, by Definition 17, the subordination result holds true if

$$
\left\{\frac{\Psi(\lambda, \gamma, k, 2)}{2[1-\gamma+\Psi(\lambda, \gamma, k, 2)]}\right\}_{n=1}^{\infty}
$$

is a subordinating factor sequence, with $a_{1}=1$. In view of Lemma 18, this is equivalent to the following inequality:

$$
\operatorname{Re}\left\{1+\sum_{n=1}^{\infty} \frac{\Psi(\lambda, \gamma, k, 2)}{[1-\gamma+\Psi(\lambda, \gamma, k, 2)]} a_{n} z^{n}\right\}>0, \quad z \in U .
$$

By noting the fact that $\Psi(\lambda, \gamma, k, n) /(1-\gamma)$ is increasing function for $n \geq 2$ and in particular

$$
\frac{\Psi(\lambda, \gamma, k, 2)}{1-\gamma} \leq \frac{\Psi(\lambda, \gamma, k, n)}{1-\gamma}, \quad n \geq 2,
$$


therefore, for $|z|=r<1$, we have

$$
\begin{gathered}
\operatorname{Re}\left\{1+\frac{\Psi(\lambda, \gamma, k, 2)}{[1-\gamma+\Psi(\lambda, \gamma, k, 2)]} \sum_{n=1}^{\infty} a_{n} z^{n}\right\} \\
=\operatorname{Re}\left\{1+\frac{\Psi(\lambda, \gamma, k, 2)}{[1-\gamma+\Psi(\lambda, \gamma, k, 2)]} z\right. \\
\left.+\frac{\sum_{n=2}^{\infty} \Psi(\lambda, \gamma, k, 2) a_{n} z^{n}}{[1-\gamma+\Psi(\lambda, \gamma, k, 2)]}\right\} \\
\geq 1-\frac{\Psi(\lambda, \gamma, k, 2)}{[1-\gamma+\Psi(\lambda, \gamma, k, 2)]} r \\
-\frac{\sum_{n=2}^{\infty} \Psi(\lambda, \gamma, k, n)\left|a_{n}\right| r^{n}}{[1-\gamma+\Psi(\lambda, \gamma, k, 2)]} \\
>0, \quad|z|=r<1,
\end{gathered}
$$

where we have also made use of the assertion (17) of Lemma 8. This evidently proves the inequality (51) and hence also the subordination result (47) asserted by Lemma 8 .

The inequality (48) follows from (47) by taking

$$
g(z)=\frac{z}{1-z}=z+\sum_{n=2}^{\infty} z^{n} \in C .
$$

Next we consider the function

$$
F(z):=z-\frac{1-\gamma}{\Psi(\lambda, \gamma, k, 2)} z^{2}
$$

where $0 \leq \gamma<1, k \geq 0,0 \leq \lambda<1$, and $\Psi(\lambda, \gamma, k, 2)$ is given by (25). Clearly $F \in \mathscr{M}_{q}(\lambda, \gamma, k)$. For this function (47) becomes

$$
\frac{\Psi(\lambda, \gamma, k, 2)}{2[1-\gamma+\Psi(\lambda, \gamma, k, 2)]} F(z) \prec \frac{z}{1-z} .
$$

It is easily verified that

$$
\min \left\{\operatorname{Re}\left(\frac{\Psi(\lambda, \gamma, k, 2)}{2[1-\gamma+\Psi(\lambda, \gamma, k, 2)]} F(z)\right)\right\}=-\frac{1}{2}, \quad z \in U
$$

This shows that the constant $\Psi(\lambda, \gamma, k, 2) / 2[1-\gamma+\Psi(\lambda, \gamma, k$, $2)$ ] cannot be replaced by any larger one.

\section{Partial Sums}

For a function $f \in A$ given by (1) Silverman [12] and Silvia [13] investigated the partial sums $f_{1}$ and $f_{m}$ defined by

$$
f_{1}(z)=z, \quad f_{m}(z)=z+\sum_{n=2}^{m} a_{n} z^{n}, \quad(m=2,3, \ldots) .
$$

We consider in this section partial sums of functions in the class $\mathscr{M}_{q}(\lambda, \gamma, k)$ and obtain sharp lower bounds for the ratios of real part of $f$ to $f_{m}(z)$ and $f^{\prime}$ to $f_{m}^{\prime}$.
Theorem 20. Let a function $f$ of the form (1) belong to the class $\mathscr{M}_{q}(\lambda, \gamma, k)$ and satisfy the condition (17). Then

$$
\begin{aligned}
& \operatorname{Re}\left\{\frac{f(z)}{f_{m}(z)}\right\} \geq 1-\frac{1}{\mathscr{C}_{m+1}}, \quad z \in U, m \in N, \\
& \operatorname{Re}\left\{\frac{f_{m}(z)}{f(z)}\right\} \geq \frac{\mathscr{C}_{m+1}}{1+\mathscr{C}_{m+1}}, \quad z \in U, m \in N,
\end{aligned}
$$

where

$$
\mathscr{C}_{n}:=\frac{n[(1+n \lambda-\lambda)(1+k)-n(\gamma+k)]}{1-\gamma} \Phi_{q}(n, \mu) .
$$

Proof. By (61) it is not difficult to verify that

$$
\mathscr{C}_{n+1}>\mathscr{C}_{n}>1, \quad n=2,3, \ldots
$$

Thus by Lemma 8 we have

$$
\sum_{n=2}^{m}\left|a_{n}\right|+\mathscr{C}_{m+1} \sum_{n=m+1}^{\infty}\left|a_{n}\right| \leq \sum_{n=2}^{\infty} \mathscr{C}_{n}\left|a_{n}\right| \leq 1 .
$$

Setting

$$
\begin{aligned}
g(z) & =\mathscr{C}_{m+1}\left\{\frac{f(z)}{f_{m}(z)}-\left(1-\frac{1}{\mathscr{C}_{m+1}}\right)\right\} \\
& =1+\frac{\mathscr{C}_{m+1} \sum_{n=m+1}^{\infty} a_{n} z^{n-1}}{1+\sum_{n=2}^{m} a_{n} z^{n-1}},
\end{aligned}
$$

it suffices to show that

$$
\Re(g(z)) \geq 0, \quad z \in U .
$$

Applying (63), we find that

$$
\begin{aligned}
\left|\frac{g(z)-1}{g(z)+1}\right| & \leq \frac{\mathscr{C}_{m+1} \sum_{n=m+1}^{\infty}\left|a_{n}\right|}{2-2 \sum_{n=2}^{m}\left|a_{n}\right|-\mathscr{C}_{m+1} \sum_{n=m+1}^{\infty}\left|a_{n}\right|} \\
& \leq 1, \quad z \in U,
\end{aligned}
$$

which readily yields the assertion (59) of Theorem 20. In order to see that

$$
f(z)=z+\frac{z^{m+1}}{\mathscr{C}_{m+1}}, \quad z \in U
$$

gives the sharp result, we observe that for $z=r e^{i \pi / m}$ we have

$$
\frac{f(z)}{f_{m}(z)}=1+\frac{z^{m}}{\mathscr{C}_{m+1}} \stackrel{z \rightarrow 1^{-}}{\longrightarrow} 1-\frac{1}{\mathscr{C}_{m+1}} .
$$

Similarly, if we take

$$
\begin{aligned}
h(z) & =\left(1+\mathscr{C}_{m+1}\right)\left\{\frac{f_{m}(z)}{f(z)}-\frac{\mathscr{C}_{m+1}}{1+\mathscr{C}_{m+1}}\right\} \\
& =1-\frac{\left(1+\mathscr{C}_{n+1}\right) \sum_{n=m+1}^{\infty} a_{n} z^{n-1}}{1+\sum_{n=2}^{\infty} a_{n} z^{n-1}}, \quad z \in U,
\end{aligned}
$$


and making use of (63), we can deduce that

$$
\begin{aligned}
\left|\frac{h(z)-1}{h(z)+1}\right| & \leq \frac{\left(1+\mathscr{C}_{m+1}\right) \sum_{n=m+1}^{\infty}\left|a_{n}\right|}{2-2 \sum_{n=2}^{m}\left|a_{n}\right|-\left(1-\mathscr{C}_{m+1}\right) \sum_{n=m+1}^{\infty}\left|a_{n}\right|} \\
& \leq 1, \quad z \in U,
\end{aligned}
$$

which leads us immediately to the assertion (60) of Theorem 20. The bound in (60) is sharp for each $m \in N$ with the extremal function $f$ given by (67), and the proof is complete.

Theorem 21. Let a function $f$ of the form (1) belong to the class $\mathscr{M}_{q}(\lambda, \gamma, k)$ and satisfy the condition (17). Then

$$
\begin{gathered}
\operatorname{Re}\left\{\frac{f^{\prime}(z)}{f_{m}^{\prime}(z)}\right\} \geq 1-\frac{m+1}{\mathscr{C}_{m+1}}, \\
\operatorname{Re}\left\{\frac{f_{m}^{\prime}(z)}{f^{\prime}(z)}\right\} \geq \frac{\mathscr{C}_{m+1}}{m+1+\mathscr{C}_{m+1}},
\end{gathered}
$$

where $\mathscr{C}_{m}$ is defined by (61)

Proof. By setting

$$
\begin{array}{r}
g(z)=\mathscr{C}_{m+1}\left\{\frac{f^{\prime}(z)}{f_{m}^{\prime}(z)}-\left(1-\frac{m+1}{\mathscr{C}_{m+1}}\right)\right\}, \quad z \in U, \\
h(z)=\left[(m+1)+\mathscr{C}_{m+1}\right]\left\{\frac{f_{m}^{\prime}(z)}{f^{\prime}(z)}-\frac{\mathscr{C}_{m+1}}{m+1+\mathscr{C}_{m+1}}\right\}, \\
z \in U,
\end{array}
$$

the proof is analogous to that of Theorem 20, and we omit the details.

\section{Integral Means}

In [1], Silverman found that the function $f_{2}(z)=z-\left(z^{2} / 2\right)$ is often extremal over the family $\mathscr{T}$. He applied this function to resolve his integral means inequality, conjectured in [14] and settled in [15], that

$$
\int_{0}^{2 \pi}\left|f\left(r e^{i \theta}\right)\right|^{\eta} d \theta \leq \int_{0}^{2 \pi}\left|f_{2}\left(r e^{i \theta}\right)\right|^{\eta} d \theta
$$

for all $f \in \mathscr{T}, \eta>0$, and $0<r<1$. In [15], he also proved his conjecture for the subclasses $\mathscr{T}^{*}(\gamma)$ the class of starlike functions and $C(\gamma)$ the class of convex functions with negative coefficients.

We recall the following lemma to prove our result on integral means inequality.

Lemma 22 (see [11]). If the functions $f$ and $g$ are analytic in $U$ with $g<f$, then for $\eta>0$, and $0<r<1$,

$$
\int_{0}^{2 \pi}\left|g\left(r e^{i \theta}\right)\right|^{\eta} d \theta \leq \int_{0}^{2 \pi}\left|f\left(r e^{i \theta}\right)\right|^{\eta} d \theta .
$$

Applying Lemmas 22 and 8 and Theorem 12, we prove the Silverman's conjecture for the functions in the family $\mathscr{M}_{q}(\lambda, \gamma, k)$ by using known procedures.

Theorem 23. Suppose that $f \in \mathscr{M}_{q}(\lambda, \gamma, k), \eta>0,0 \leq \lambda<1$, $0 \leq \gamma<1, k \geq 0$, and $f_{2}(z)$ is defined by

$$
f_{2}(z)=z-\frac{1-\gamma}{\Psi(\lambda, \gamma, k, 2)} z^{2}
$$

where $\Psi(\lambda, \gamma, k, 2)$ is defined in (25). Then for $z=r e^{i \theta}, 0<r<$ 1 , we have

$$
\int_{0}^{2 \pi}|f(z)|^{\eta} d \theta \leq \int_{0}^{2 \pi}\left|f_{2}(z)\right|^{\eta} d \theta .
$$

Concluding Remarks. In fact, by suitably specializing the values of $\lambda$ and $k$, the results presented in this paper would find further applications for the class of univalent starlike functions with negative coefficients stated in Examples 4 and 5 of Section 1 .

\section{References}

[1] H. Silverman, "Univalent functions with negative coefficients," Proceedings of the American Mathematical Society, vol. 51, pp. 109-116, 1975.

[2] S. D. Purohit and R. K. Raina, "Certain subclasses of analytic functions associated with fractional q-calculus operators," Mathematica Scandinavica, vol. 109, no. 1, pp. 55-70, 2011.

[3] G. Gasper and M. Rahman, Basic Hypergeometric Series, vol. 35 of Encyclopedia of Mathematics and Its Applications, Cambridge University Press, Cambridge, UK, 1990.

[4] Y. C. Kim and H. M. Srivastava, "Fractional integral and other linear operators associated with the Gaussian hypergeometric function," Complex Variables. Theory and Application, vol. 34, no. 3, pp. 293-312, 1997.

[5] A. W. Goodman, "On uniformly starlike functions," Journal of Mathematical Analysis and Applications, vol. 155, no. 2, pp. 364370, 1991.

[6] F. Rønning, "Uniformly convex functions and a corresponding class of starlike functions," Proceedings of the American Mathematical Society, vol. 118, no. 1, pp. 189-196, 1993.

[7] K. G. Subramanian, G. Murugusundaramoorthy, P. Balasubrahmanyam, and H. Silverman, "Subclasses of uniformly convex and uniformly starlike functions," Mathematica Japonica, vol. 42, no. 3, pp. 517-522, 1995.

[8] A. W. Goodman, "Univalent functions and nonanalytic curves," Proceedings of the American Mathematical Society, vol. 8, pp. 598-601, 1957.

[9] S. Ruscheweyh, "Neighborhoods of univalent functions," Proceedings of the American Mathematical Society, vol. 81, no. 4, pp. 521-527, 1981.

[10] H. S. Wilf, "Subordinating factor sequences for convex maps of the unit circle," Proceedings of the American Mathematical Society, vol. 12, pp. 689-693, 1961.

[11] J. E. Littlewood, "On inequalities in the theory of functions," Proceedings of the London Mathematical Society, vol. 23, no. 1, pp. 481-519, 1925.

[12] H. Silverman, "Partial sums of starlike and convex functions," Journal of Mathematical Analysis and Applications, vol. 209, no. 1, pp. 221-227, 1997. 
[13] E. M. Silvia, "On partial sums of convex functions of order $\alpha$," Houston Journal of Mathematics, vol. 11, no. 3, pp. 397-404, 1985.

[14] H. Silverman, "A survey with open problems on univalent functions whose coefficients are negative," The Rocky Mountain Journal of Mathematics, vol. 21, no. 3, pp. 1099-1125, 1991.

[15] H. Silverman, "Integral means for univalent functions with negative coefficients," Houston Journal of Mathematics, vol. 23, no. 1, pp. 169-174, 1997. 


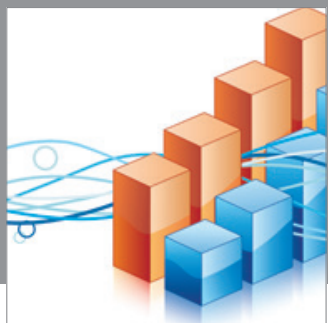

Advances in

Operations Research

mansans

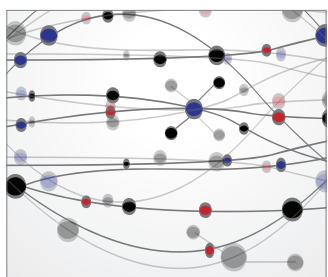

The Scientific World Journal
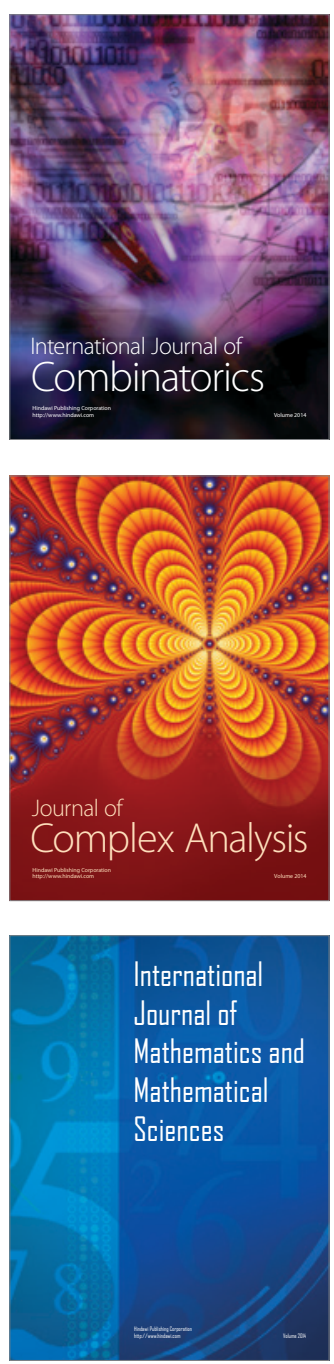
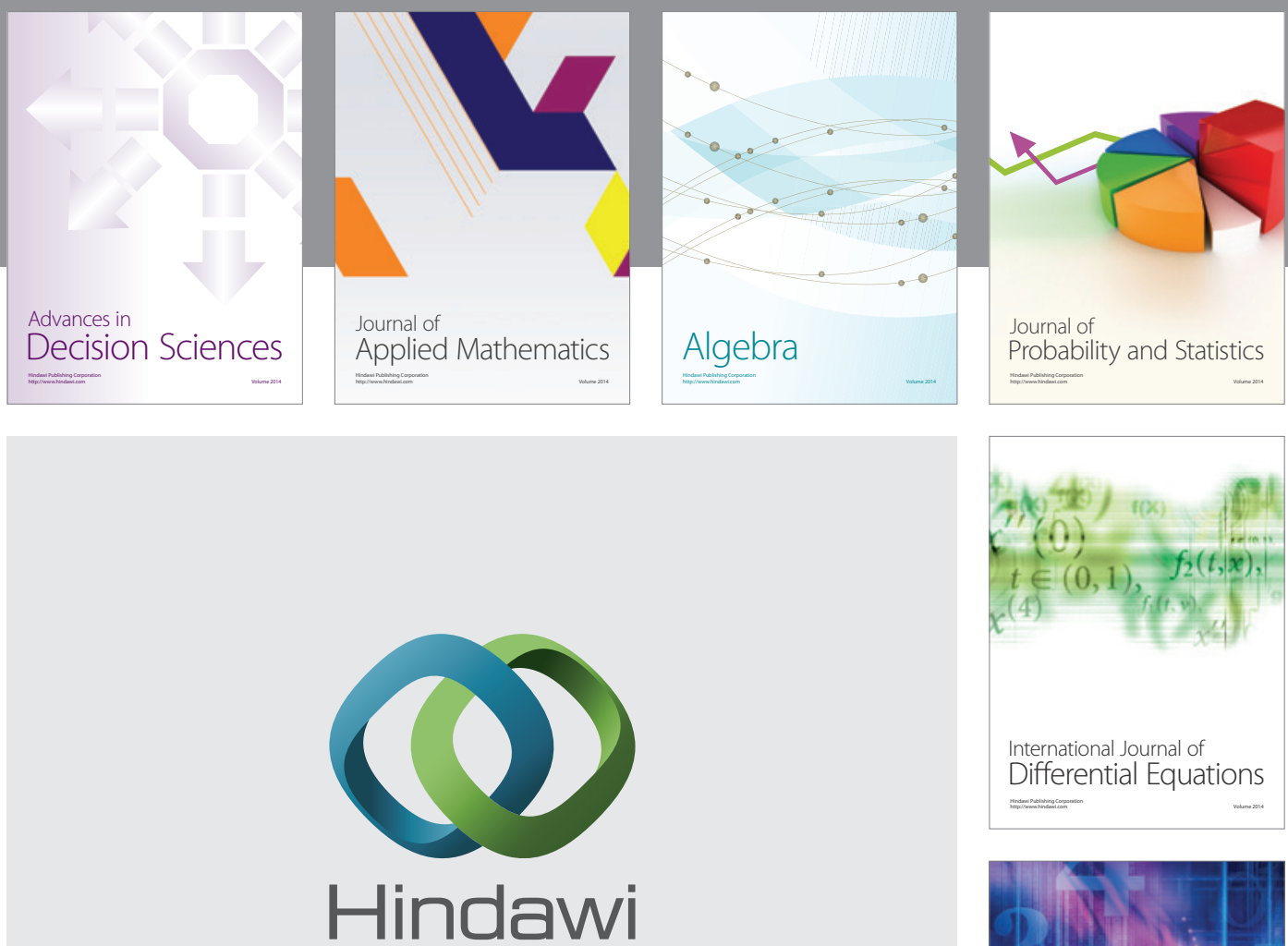

Submit your manuscripts at http://www.hindawi.com
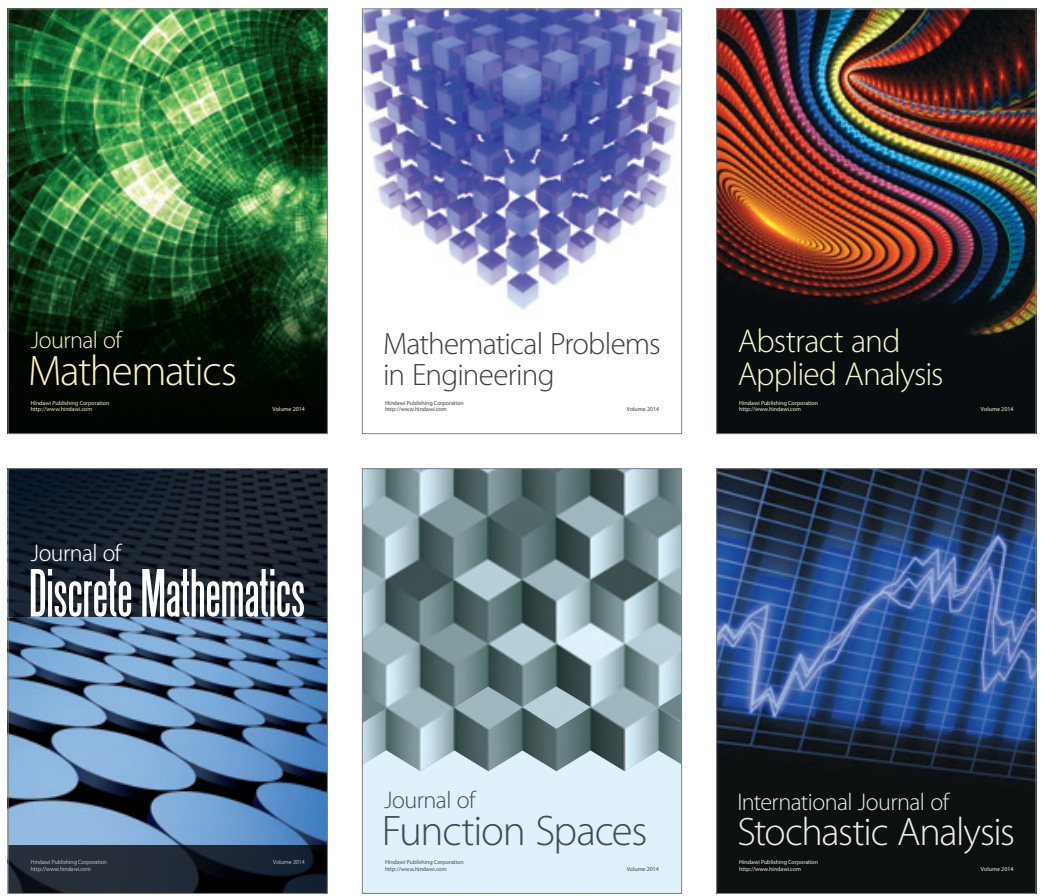

Journal of

Function Spaces

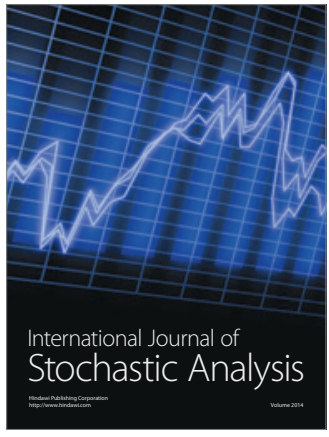

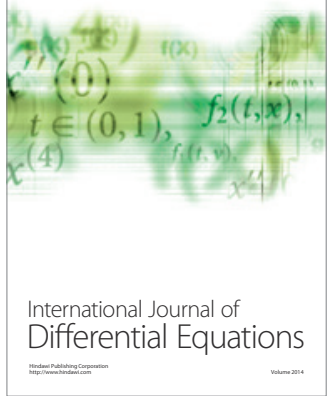
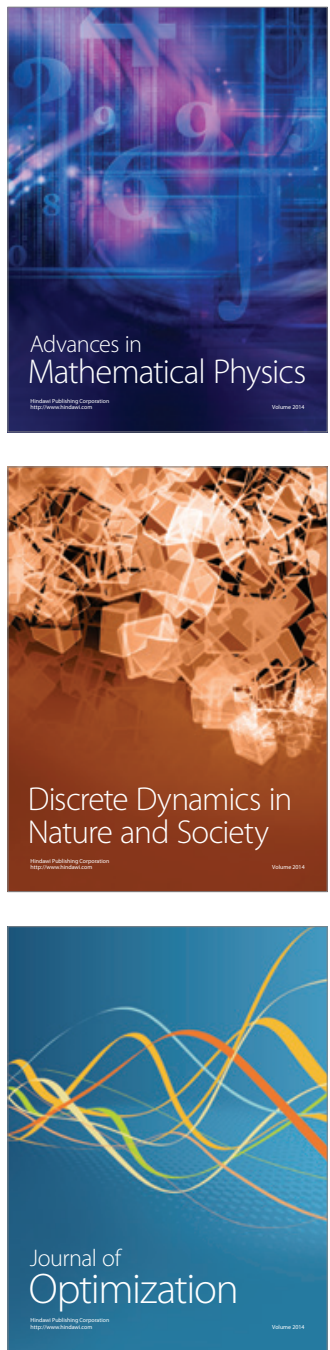